\title{
Tomotherapy for neurofibromatosis Type 2: case report and review of the literature
}

\author{
${ }^{1} \mathrm{H}-\mathrm{H}$ LEE, MD, ${ }^{1,2} \mathrm{~S}-\mathrm{L}$ LIAN, MD, PhD, ${ }^{1,2} \mathrm{C}-J$ HUANG, MD and ${ }^{1,2} \mathrm{M}-\mathrm{Y}$ HUANG, MD \\ ${ }^{1}$ Division of Radiation Oncology, Department of Radiation Oncology, Kaohsiung Medical University Hospital, Kaohsiung \\ Medical University, Kaohsiung, Taiwan, and ${ }^{2}$ Department of Radiation Oncology, Faculty of Medicine, College of \\ Medicine, Kaohsiung Medical University, Kaohsiung, Taiwan
}

\begin{abstract}
Neurofibromatosis 2 (NF2) results in multiple central nervous system tumours. In this case report, the patient has one vestibular schwannoma, one trigeminal schwannoma and two meningiomas developed before the age of 30 . Aiming to treat three targets at one fraction with minimal interaction and overlapping doses to normal tissue, the sophisticated equipment of tomotherapy was utilised for frameless stereotaxy; tomotherapy delivered intensity-modulated, rotational radiation therapy using a fan-beam delivery. Daily CT scans with the inbuilt CT scanner were also performed as part of the image-guided radiotherapy. The course of fractionated stereotactic radiotherapy consisted of eight fractions given three times per week with an overall treatment time of 17 days. For the meningioma over left parietal vertex, 4.5 Gy per fraction was given at $36 \mathrm{~Gy} / 8 \mathrm{Fr} / 17$ days. For the meningioma over anterior cerebral falx, 4 Gy per fraction was given at $32 \mathrm{~Gy} / 8 \mathrm{Fr} / 17$ days. For the two schwannomas as one target, $5 \mathrm{~Gy}$ per fraction was given at $40 \mathrm{~Gy} / 8 \mathrm{Fr} / 17$ days. The acute effect of the treatment was alopecia and mild headache. Subsequent follow-up confirmed clinical improvement. This is the first reported case of clinical experience with tomotherapy in the management of NF2.
\end{abstract}

Received 29 December 2008

Revised 25 March 2009 Accepted 21 April 2009

DOI: $10.1259 /$ bjr/16531514

(C) 2010 The British Institute of Radiology
Neurofibromatosis 2 (NF2) is a slow-growing and nonmalignant inherited cancer syndrome with an estimated incidence of 1:40 000 [1]. The diagnosis is often made in the second or third decade of life. All patients have been found to have some mutation of the same gene located on chromosome 22. Statistically, one-half of cases are inherited and one-half are the result of de novo mutations. Inactivation of the NF2 gene and loss of expression of its products, merlin or schwannomin, has been reported for all NF2-associated tumours [2]. The autosomal dominant genetic predisposition to the development of various neural crest cell neoplasms is characteristic [3]. The principal treatment for symptomatic NF2 in our institute consists of surgical intervention accompanied by either radiotherapy for residual tumours or observation. Radiotherapy can be reserved for recurrence. There is no therapy for the underlying disorder of cell function caused by the genetic mutation.

Multiple central nervous system (CNS) tumours are often the consequence of the development of NF2. In most applications of radiotherapy, including arc therapy, stereotactic radiosurgery and Gamma Knife, different dosage for different lesions in one single treatment course is not achievable. By contrast, tomotherapy is able

Address correspondence to: Dr Shi-Long Lian, Department of Radiation Oncology, Kaohsiung Medical University Hospital, 100 Shih-Chuan 1st Road, Kaohsiung 807, Taiwan. E-mail: shloli@cc.kmu.edu.tw to carry out the task of giving different doses to different lesions with minimal overlapping to reduce significant sequelae [4-6].

\section{Case report}

A 37-year-old woman presented to our Radiation Oncology outpatient department with increased frequency and intensity of headache, vertigo, unsteadiness and left facial numbness. Tracing her history back 10 years, when she initially developed tinnitus along with hearing impairment, MRI of the head then revealed a mass in the left cerebellopontine angle (CPA) (Figure 1a) and a durally based mass in the anterior aspect of interhemisphere fissure characteristic of meningioma (Figure 1b). The patient underwent left CPA tumour excision via left occipital craniectomy in 1998. The histopathological report revealed two schwannomas originating from the fifth cranial nerve and the eighth cranial nerve, respectively.

MRI subsequently revealed multiple neoplastic lesions at different brain sites. We informed the patient about surgical intervention but she refused a second craniectomy.

When treatment was selected there were only two viable alternatives: microsurgical removal or radiotherapy. Owing to her reluctance to have an operation, the patient was referred to the Department of Radiation Oncology. Upon presentation at the age of 37 years, she 


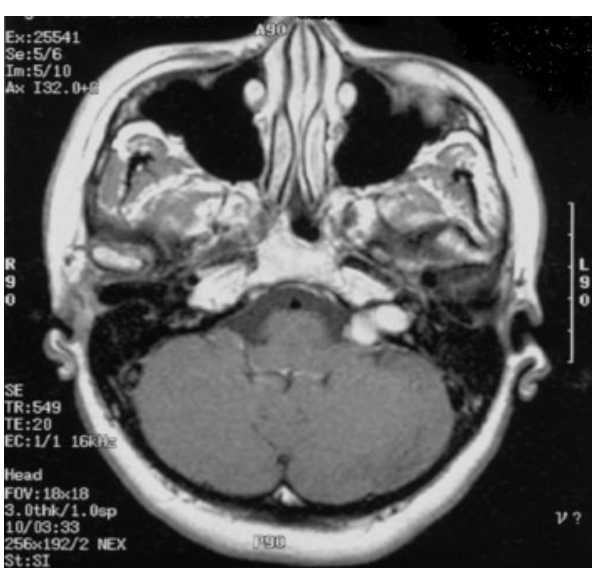

(a)

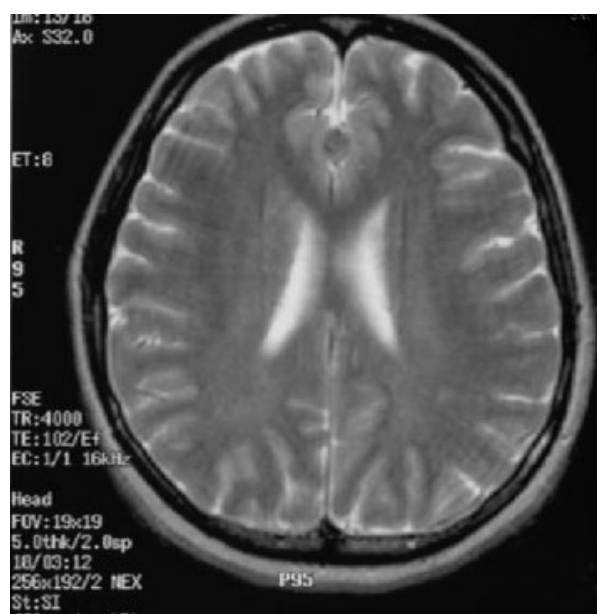

(b)

Figure 1. Pre-operative MRI of the patient at the age of 28 years in 1998. (a) A soft tissue nodule with heterogeneous signal intensity was apparent within the widened left internal acoustic canal; the enhancement was strong and homogenous in the left cerebellopontine angle. (b) A well-circumscribed meningioma in the anterior aspect of interhemisphere fissure.

had the stigmata of NF2 with two meningiomas and two schwannomas from distinct cranial nerves (Figure 2). MRI demonstrated a CPA tumour indenting and deforming the brain stem with life-threatening potential.

According to the patient, there was complete hearing loss on the operative side, left facial paralysis and hypesthesia. Recently she had been suffering from excruciating headache that interfered with her daily activity. On neurological examination, there were palsies of the left fifth, seventh and eighth cranial nerves. There was no skin manifestation, no prior history of head or neck irradiation or any previous exposure to toxins.

Tomotherapy to three locations at a time was exerted for tumour control while avoiding the risks of surgery. The two schwannomas derived from trigeminal and vestibular cranial nerves formed one mass located in the CPA with a volume of $39.16 \mathrm{cc}$ (Figure 2). The volume of the meningioma over left parietal vertex was $16.1 \mathrm{cc}$ and that of the meningioma over anterior cerebral falx was $7.78 \mathrm{cc}$.

Hi-Art helical tomotherapy (TomoTherapy, Inc., Madison, WI) was used to deliver frameless stereotaxy. The gross tumour volume (GTV) was contoured on contrast CT. The field width was $2.5 \mathrm{~cm}$, the pitch was 0.3 and the planning modulation factor was set to be 2.5 (the actual modulation factor was 1.658). The patient was immobilised with a thermoplastic mask. For the meningioma over left parietal vertex as target 1, 4.5 Gy per fraction was given at $36 \mathrm{~Gy}$ in eight fractions over 17 days (36 Gy/8 Fr/17 days). For the meningioma over anterior cerebral falx as target 2, 4 Gy per fraction was given at $32 \mathrm{~Gy} / 8 \mathrm{Fr} / 17$ days. For the CPA schwannomas as target 3, 5 Gy per fraction was given at $40 \mathrm{~Gy} /$ $8 \mathrm{Fr} / 17$ days. Higher doses were given to larger lesions; for the recurrent schwannomas, a high dose was prescribed to curb the disease progression. Dose was prescribed to the planning targets with no volumetric expansion. Of the target volume, 95\% received at least the prescribed dose. In arriving at an optimal plan, great care was taken to respect the brain stem tolerance. Our goal was to make the maximal dose less than $28 \mathrm{~Gy}$ and such a constraint resulted in a less acute curve shown on the dose-volume histogram (DVH) (Figure 3). The treatment duration per fraction was $617.3 \mathrm{~s}$. Fractionated stereotactic radiotherapy (FSRT) was administered three times a week with eight fractions in total. The overall treatment time was 17 days.

The acute effects of the treatment were hair loss along with mild headache and one episode of vomiting during the treatment course. General malaise and occasional nausea also developed. There was no change noted in the neurological exam. Subsequent follow-up confirmed the alleviation of headache that allowed the patient to return to her previous employment after treatment. Figure 4 shows an MRI 1 year post treatment.

\section{Discussion}

There has been some debate that the current diagnostic criteria for NF2 might be too narrow. Baser et al [7] appraised four sets of diagnostic systems that vary for people without bilateral vestibular schwannoma, which are pathognomonic for NF2. Their study in 2006 concluded that overall, and at the time of the most recent clinical evaluation, the Manchester criteria are the most sensitive [7].

The diagnostic Manchester criteria for NF2 are met when an individual has one of the following: bilateral vestibular schwannomas; a first-degree relative with NF2 and unilateral vestibular schwannoma or any two of meningioma, schwannoma, glioma, neurofibroma or posterior subcapsular lenticular opacities; unilateral vestibular schwannoma and any two of meningioma, schwannoma, glioma, neurofibroma or posterior subcapsular lenticular opacities; multiple meningiomas (two or more) and unilateral vestibular schwannoma or any two of schwannoma, glioma, neurofibroma or cataract [8]. The clinical diagnosis of NF2 is a continuous process that can change as new tumours arise [9].

Although schwannoma is sporadically manifested as a single benign neoplasm, the presence of multiple schwannomas in one patient is usually indicative of NF2 [10]. In a study by Baser and colleagues, $41 \%$ of 


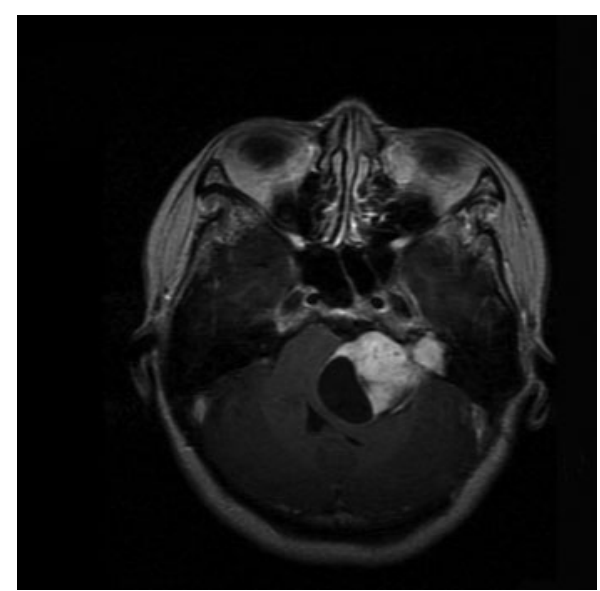

(a)

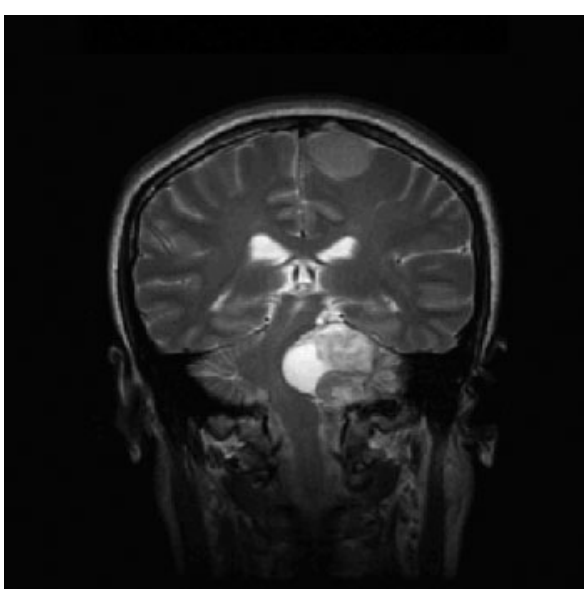

(c)

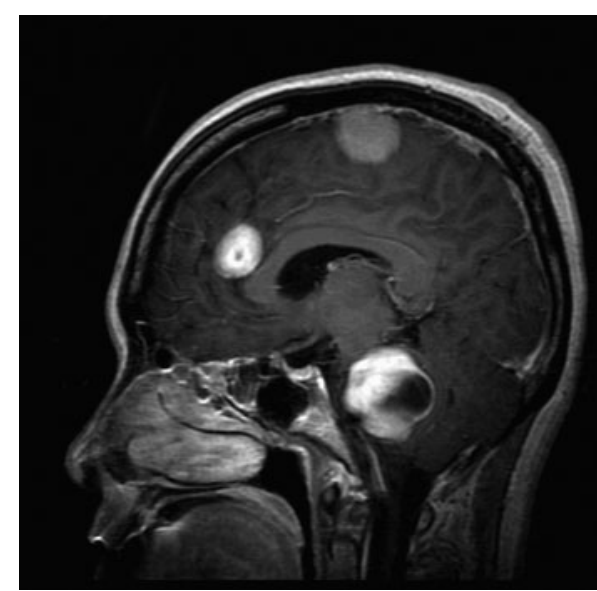

(b)

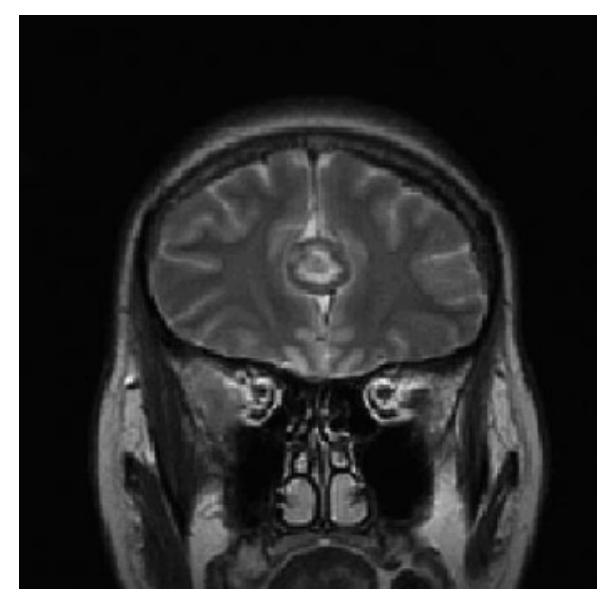

(d)

Figure 2. MRI of the patient at 37 years of age in 2007. (a) Axial view ( $T_{1}$ flair with contrast enhancement). (b) Sagittal view ( $T_{1}$ flair with contrast enhancement). Recurrent left cerebellopontine and intracanalicular acoustic neuroma of size $3.48 \times 3.82 \times 3.47 \mathrm{~cm}$ encompassing solid and cystic parts. There was compression of brain stem. (c) Coronal view (fast-spin echo (FSE) $T_{2}$ weighted). Enlargement of meningioma of size $2.82 \times 3.09 \times 2.25 \mathrm{~cm}$ in left parietal vertex showed homogeneous $T_{2}$ weighted high signal and homogeneous enhancement. (d) Coronal view (FSE $T_{2}$ weighted). Enlargement of meningioma with the greatest diameter of $1.8 \mathrm{~cm}$ in anterior cerebral falx showed a rim with low $T_{2}$ weighted signal intensity and intermediate $T_{2}$ weighted signal intensity indicating calcification.

individuals with clinical information in the UK NF2 registry did not have bilateral vestibular schwannoma at initial assessment [7]. Without family history or even the hallmark of bilateral vestibular schwannoma, the patient described here had two schwannomas and two meningiomas before the age of 30 and met the Manchester criteria of NF2. In recent follow-up MRI, another meningioma has arisen along the left aspect of cerebellar tentorium with homogeneous enhancement (Figure 4).

In general, vestibular schwannomas should be removed when still small for best preservation of facial nerve function and hearing, but NF2 vestibular schwannomas seem to be more infiltrative to adjacent nerves than non-NF2 tumours [11-13]. The first operation that our patient underwent at the age of 28 left behind not only residual tumour, but also sequelae of left hearing impairment and facial hypaesthesia.

Our patient, as well as being the first reported case of clinical experience with tomotherapy in the management of NF2, also highlights an intriguing link between the application of tomotherapy and multiple CNS tumours.
Tomotherapy, introduced in 2004, is able to treat a range of neoplasms using a single helical beam with 360 degrees of rotation. Different doses can be applied to each of the different lesions at a time.

With brain stem distortion, these locally aggressive yet benign neoplasms can be critical. Our therapeutic principle for NF2 is to selectively irradiate all sharply defined targets at one time, while largely sparing the surrounding normal tissue such as brain stem, spinal cord and eyes. All lesions were irradiated with the smallest overlaps of irradiation fields within one treatment course of FSRT.

There were eight fractions in our treatment planning and each was given every other day besides weekends as hypofractionated regimen. Several studies have reported similar control rates achieved by FSRT without the high morbidity associated with single-dose radiosurgery [14]. Stereotactic radiosurgery (SRS) is associated with an increased toxicity to the facial and trigeminal nerves, as compared with FSRT. With FSRT, rates of treatment-related toxicity to the facial nerve of $0-3 \%$ can be found in the 


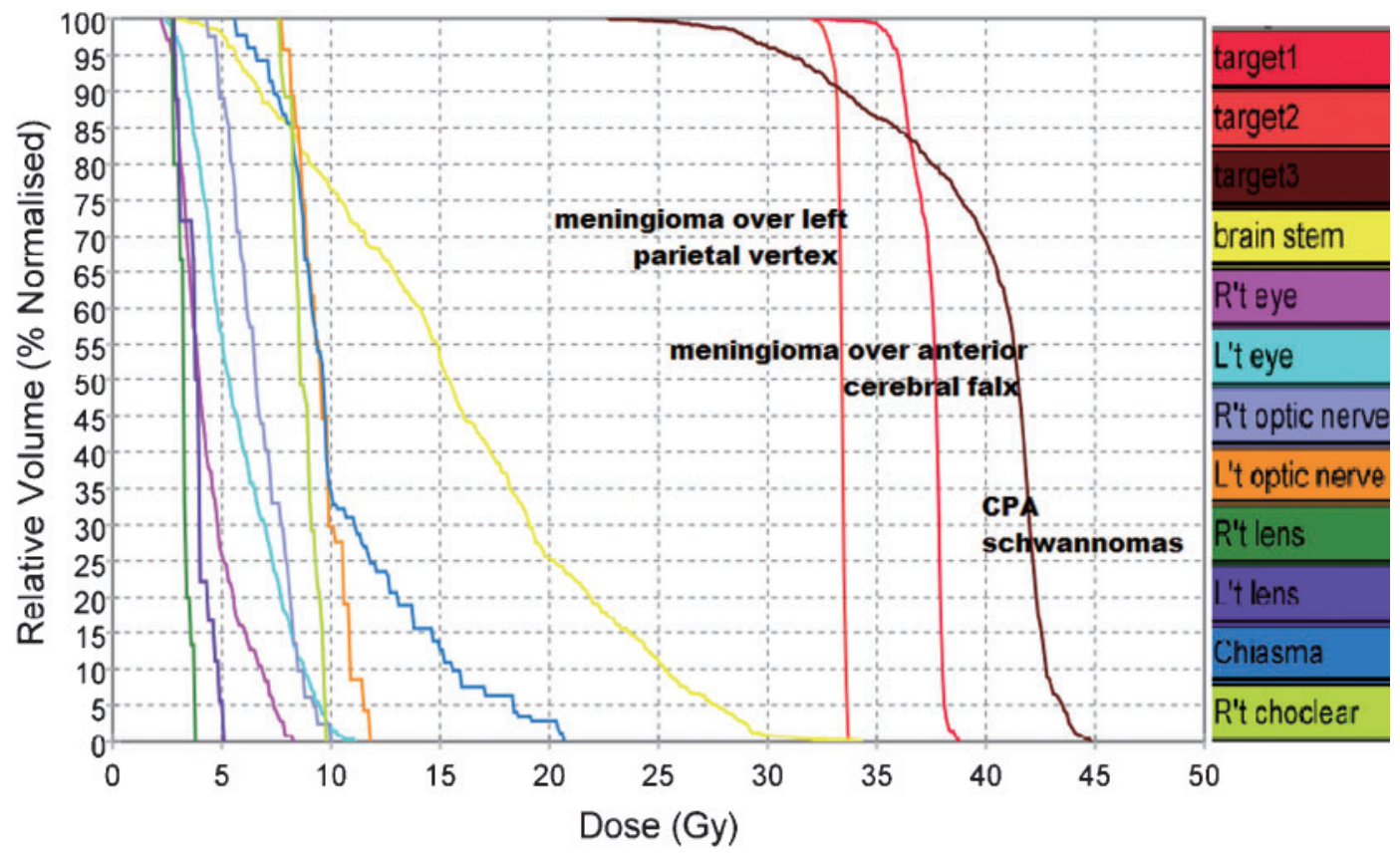

Figure 3. Dose-volume histogram with tomotherapy treatment planning (Hi-ART, version 2.2.4.1).

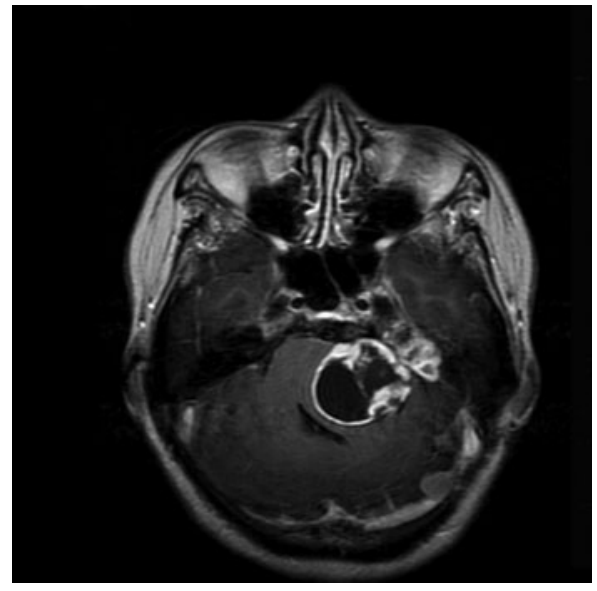

(a)

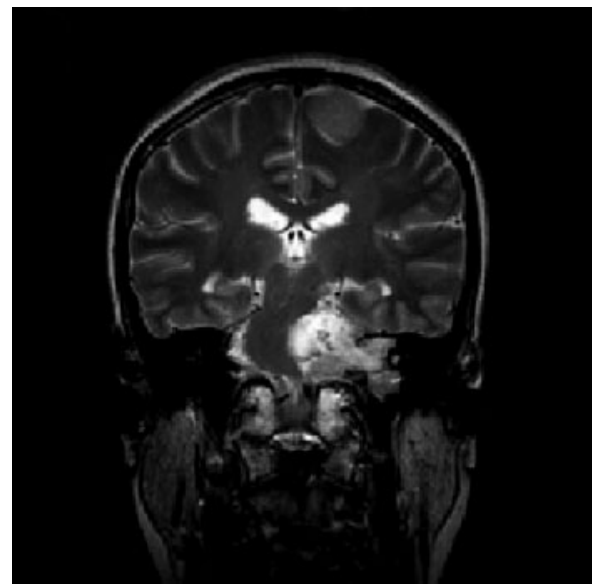

(c)

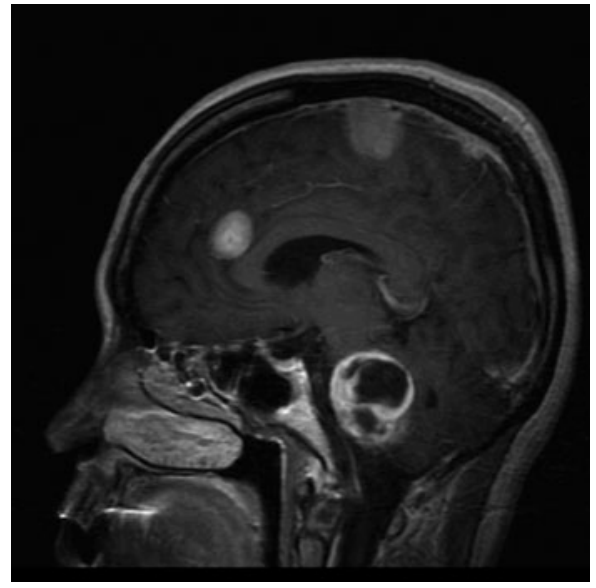

(b)

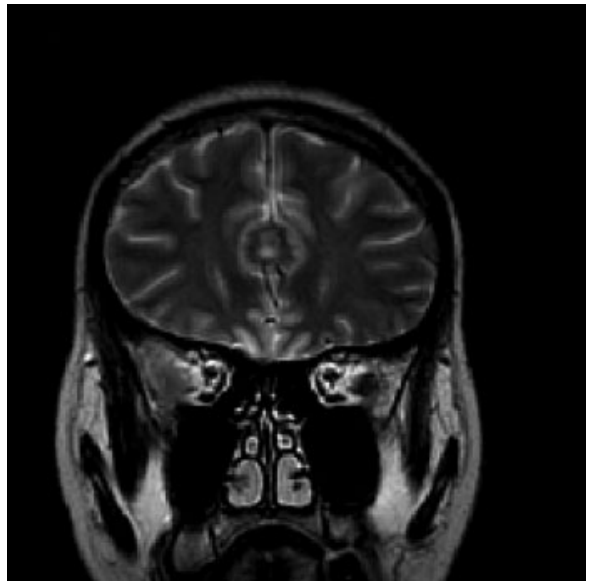

(d)

Figure 4. MRI of the patient at 38 years of age in 2008, 1 year after FSRT. (a) Axial view ( $T_{1}$ flair with contrast enhancement). A new meningioma has arisen along the left aspect of cerebellar tentorium with homogeneous enhancement. (b) Sagittal view $\left(T_{1}\right.$ flair with contrast enhancement). Two meningiomas and one cerebellopontine angle (CPA) tumour post radiotherapy are shown. (c) Coronal view (fast-spin echo (FSE) $T_{2}$ weighted). The meningioma in left parietal vertex and the CPA tumour post radiotherapy are shown. (d) Coronal view (FSE $T_{2}$ weighted). The meningioma in anterior cerebral falx post radiotherapy is shown. 
literature [15-22]. With microsurgery, the rate of cranial nerve toxicity depends strongly on the size of the vestibular schwannoma. In patients with large tumours, facial palsy rates of $40-80 \%$ could be found $[23,24]$. Treatment of acoustic neuroma with SRS might lead to facial nerve toxicity rates of $0-23 \%$, as reported in different studies [25].

\section{Conclusion}

The reported case presents unique opportunities to gain insights into the application of radiotherapy associated with multiple targeting. Obvious benefits of tomotherapy are its non-invasive nature and the application of high therapeutic doses to different lesions with the least overlap. However, large penumbra can bear the theoretical risk of a second malignancy and relevant precautions should be discussed with the patient. Tomotherapy is a relatively new addition to the armamentarium of cancer therapeutics that provides symptom control and stabilisation of disease progression.

\section{References}

1. Pollack IF, Mulvihill JJ. Neurofibromatosis 1 and 2. Brain Pathol 1997;7:823-36.

2. Gutmann DH. The neurofibromatoses: when less is more. Hum Mol Genet 2001;10:747-55.

3. Arnold SR, Baker CC, Gilbert-Barness E. Pathological case of the month. Arch Pediatr Adolesc Med 2000;154:961-2.

4. Tomita N, Kodaira T, Tachibana H, Nakamura T, Nakahara $\mathrm{R}$, Inokuchi $\mathrm{H}$, et al. Helical tomotherapy for brain metastases: dosimetric evaluation of treatment plans and early clinical results. Technology Cancer Res Treat 2008;7: $417-23$.

5. Rades D, Kueter JD, Hornung D, Veninga T, Hanssens P, Schild SE, et al. Comparison of stereotactic radiosurgery (SRS) alone and whole brain radiotherapy (WBRT) plus a stereotactic boost (WBRT + SRS) for one to three brain metastases. Strahlenther Onkol 2008;184:655-62.

6. Peñagarícano JA, Yan $Y$, Shi $C$, Linskey ME, Ratanatharathorn V. Dosimetric comparison of helical tomotherapy and gamma knife stereotactic radiosurgery for single brain metastasis. Radiat Oncol 2006;1:26.

7. Baser ME, Friedman JM, Evans DGR. Increasing the specificity of diagnostic criteria for schwannomatosis. Neurology 2006;66:730-2.

8. Evans DGR, Huson SM, Donnai D, Neary W, Blair V, Newton V, et al. A clinical study of type 2 neurofibromatosis. Q J Med 1992;84:603-18.

9. Chen AF, Samy RN, Gantz BJ. Cerebellopontine angle tumor composed of Schwann and meningeal proliferations. Arch Otolaryngol Head Neck Surg 2001;127:1385-9.

10. Ahn JY, Kwon SO, Shin MS, Shim JY, Kim OJ. A Case of multiple schwannomas of the trigeminal nerves, acoustic nerves, lower cranial nerves, brachial plexuses and spinal canal: schwannomatosis or neurofibromatosis? Yonsei Med J 2002;43:109-13.
11. Linthicum FH Jr, Brackmann DE. Bilateral acoustic tumors. A diagnostic and surgical challenge. Arch Otolaryngol 1980;106:729-33.

12. Marquet JF, Forton GE, Offeciers FE, Moeneclaey LL. The solitary schwannoma of the eighth cranial nerve. An immunohistochemical study of the cochlear nerve-tumor interface. Arch Otolaryngol Head Neck Surg 1990;116:1023-5.

13. Jaaskeliiinen J, Paetau A, Pyykko I, Blomstedt G, Palva T, Troupp $\mathrm{H}$. Interface between the facial nerve and large acoustic neurinomas. Immunohistochemical study of the cleavage plane in NF2 and non-NF2 cases. J Neurosurg 1994;80:541-7.

14. Combs SE, Volk S, Schulz-Ertner D, Huber PE, Thilmann C, Debus Jg. Management of acoustic neuromas with fractionated stereotactic radiotherapy (FSRT): long-term results in 106 patients treated in a single institution. Int J Radiat Oncol Biol Phys 2005;63:75-81.

15. Andrews DW, Suarez O, Goldman HW, Downes, B, Bednarz G, Corn BW, et al. Stereotactic radiosurgery and fractionated stereotactic radiotherapy for the treatment of acoustic schwannomas: comparative observations of 125 patients treated at one institution. Int J Radiat Oncol Biol Phys 2001;50:1265-78.

16. Varlotto JM, Shrieve DC, Alexander E III, Kooy HM, Black PM, Locffler JS. Fractionated stereotactic radiotherapy for the treatment of acoustic neuromas: preliminary results. Int J Radiat Oncol Biol Phys 1996;36:141-5.

17. Meijer OW, Vandertop WP, Baayen JC, Slotman BJ. Singlefraction vs. fractionated linac-based stereotactic radiosurgery for vestibular schwannoma: a single-institution study. Int J Radiat Oncol Biol Phys 2003;56:1390-6.

18. Maire JP, Floquet A, Darrouzet V, Guerin J, Bebear JP, Caudry M. Fractionated radiation therapy in the treatment of stage III and IV cerebello-pontine angle neurinomas: preliminary results in 20 cases. Int J Radiat Oncol Biol Phys 1992;23:147-52.

19. Lederman G, Arbit E, Lowry J. Management of acoustic neuroma. N Engl J Med 1999;340:1119-20.

20. Glass J, Silverman CL, Axelrod R, Corn BW, Andrews DW. Fractionated stereotactic radiotherapy with cis-platinum radiosensitization in the treatment of recurrent, progressive, or persistent malignant astrocytoma. Am J Clin Oncol 1997;20:226-9.

21. Combs SE, Volk S, Schulz-Ertner D, Huber PE, Thilmann C, Debus J. Management of acoustic neuromas with fractionated stereotactic radiotherapy (FSRT): long-term results in 106 patients treated in a single institution. Int J Radiat Oncol Biol Phys 2005;63:75-81.

22. Ito K, Kurita H, Sugasawa K, Mizuno M, Sasaki T. Neurootological findings after radiosurgery for acoustic neurinomas. Arch Otolaryngol Head Neck Surg 1996;122:1229-33.

23. Gormley WB, Sekhar LN, Wright DC, Kamerer D, Schessel D. Acoustic neuromas: results of current surgical management. Neurosurgery 1997;41:50-8.

24. Flickinger JC, Kondziolka D, Maitz AH, Lunsford LD. Gamma knife radiosurgery of imaging-diagnosed intracranial meningioma. Int J Radiat Oncol Biol Phys 2003;56: 801-6.

25. Combs SE, Thilmann C, Debus J, Schulz-Ertner D. Longterm outcome of stereotactic radiosurgery (SRS) in patients with acoustic neuromas. Int J Radiat Oncol Biol Phys 2006;64:1341-7. 